\title{
The Intersection of Art and Technology
}

\author{
Antonio Camurri and Gualtiero Volpe \\ Casa Paganini-InfoMus, University of Genova
}

For a long time, art and science were viewed as distant domains that were only loosely connected, but we're now witnessing more interaction between the two. This has led to an increased awareness of how art and science are indeed two different but strongly coupled aspects of human creativity, both driving innovation as art influences science and technology, and as science and technology in turn inspire art (see the two related sidebars).

Recognizing this mutually beneficial relationship, researchers at the Casa Paganini-InfoMus Research Centre (www.casapaganini.org) aim to combine scientific research in information and communications technology (ICT) with artistic and humanistic research. We carry out scientific and technological research on human-centered computing, where art and humanistic theories are a fundamental source of inspiration, capable of deep cross-fertilization with "hard" sciences and engineering. Here, we discuss some of our work, showing how our collaboration with artists informed our work on analyzing nonverbal expressive and social behavior and contributed to tools, such as the EyesWeb XMI hardware and software platform (www.infomus.org/eyesweb_ita.php), that support both artistic and scientific developments. ${ }^{1,2}$ We also sketch out how art-informed multimedia and multimodal technologies find application beyond the arts, in areas including education, cultural heritage, social inclusion, therapy, rehabilitation, and wellness.

\section{//ARTIST: Insert sidebars near here.//}

\section{Research Driven by Art}

In keeping with its mission to experiment with and establish synergies between science, technology, and the arts, the premises of Casa Paganini-InfoMus are an ancient monumental building endowed with a 230-seat auditorium and some museum rooms (see www.youtube.com/InfoMusLab for more information). The main lab room enjoys a direct view of the auditorium's stage. This configuration is paradigmatic of the interaction between artistic and scientific and technological research and enables the development of experiments, prototypes, and demonstrations in an almost realworld (ecological) scenario (see Figure 1).

Figure 1. Santa Maria delle Grazie la Nuova, premise of the Casa Paganini-InfoMus research center, is an example of innovative reuse in contemporary information society of a monumental building as a research facility on science, technology, and art. (a) The main lab room enjoys a direct view of the auditorium's stage. This configuration is paradigmatic of the interaction between (b) artistic and scientific and technological research. Here, we show an experiment on multimodal recordings (audio, video, motion capture, and physiological signals) of a string quartet.

Our research into the automated analysis of nonverbal expressive and social behavior, leading to the development of the EyesWeb platform, was heavily influenced by collaborations with composer Luciano Berio and with various artists and researchers, including Nicola Bernardini and Alvise Vidolin. The aim of the collaborations with Berio was to design and develop interactive on-stage technologies for his operas Outis (Teatro alla Scala, Milan, 1996) and Cronaca del Luogo (Salzburg Festival, 1999). EyesWeb aimed to offer synchronized recording and real-time interactive processing and rendering of multimodal data.

For Outis, we answered the following artistic research question: how might an orchestra conductor conduct and synchronize musicians with sounds electronically synthesized in real time, and with the movement of mimes on stage? We developed interactive technologies, detecting the movement of the mimes, to control in real time the electronic sounds, thus simplifying the conductor's task.

In Cronaca del Luogo, the task was more difficult. A major singer of the opera (David Moss) played a schizophrenic character at times appearing wise and calm, while other times appearing crazy, with nervous and jerky movements. We automatically measured some of his movement qualities using sensors embedded in his clothes and a flashing infrared light on his helmet, synchronized with video cameras positioned above the stage (see Figure 2). We used this information to morph the singer's voice from profound (wise) to a harsh, sharp (crazy) timbre. This experience with concrete real-world needs for multimodal analysis and mapping was of paramount importance to shape the requirements for our first publicly available version of EyesWeb. ${ }^{1}$ 
Figure 2. The sensor systems adopted for the performer David Moss in Luciano Berio's “Cronaca del Luogo" opera opening at the Salzburg Festival 1999. (a) Wireless sensors were sewn into his costume and worn in his palms. His helmet included flashing infrared LEDs, synchronized with video cameras in the theater, to track his position $(b)$ on the whole stage and to measure, in real time, details on his movement qualities, which were used to morph his voice to match character's schizophrenic behavior.

Furthermore, in our research, art didn't merely inform our requirements. It was also a valuable testbed for assessing computational models, algorithm, interfaces, and techniques. For example, Roberto Doati's musical theater work, $U n$ avatar del Diavolo [A Devil's Avatar] (performed at the Venice Biennale arts organization in Italy in 2005), was our experimental testbed to improve and evaluate multimodal acoustic interfaces exploiting sound propagation in physical objects to localize where a surface is touched and to measure the expressive qualities of such touching gestures. Surfaces of common objects belonging to the scenery on stage (a chair, an old radio, and other furniture) were used in the piece, and we could automatically distinguish, for example, between a caress, a touch with nails or fingertips, and a touch while sitting or while moving around the chair.

Ensemble musical performances and the audience experience were our testbed in the European ICT project SIEMPRE for developing and evaluating models and techniques for measuring creative social interaction between performers, and between performers and the audience, in an ecologically valid framework - that is, in a condition resembling not a laboratory experiment but rather something very near to a real music performance in a concert hall. Experiments with famous artists - such as the Quartetto di Cremona string quartet-led us to novel multimodal techniques, implemented as real-time EyesWeb software libraries, to measure nonverbal social signals, including entrainment (physical alignment between the individuals in a group) and leadership.

\section{New Ways of Creating Art}

One way in which our scientific and technological research has affected the arts is through the production of art- that is, in providing content creators (including composers, choreographers, and directors) and performers with tools for extending their artistic language and capabilities. Such an impact mainly emerges when content creators are involved in the participatory design of either an artistic or a scientific project within a multidisciplinary team. Several examples of this process are available in the history of contemporary music. In the 50s and $60 \mathrm{~s}$, thanks to available audio and electronic technologies, composers such as Luigi Nono and Karlheinz Stockhausen extended the music language by introducing the 3D spatialization of sound. Then, electroacoustic music and live electronics extended traditional orchestration techniques enabling, for example, complex transformations of sound. ${ }^{3}$

Concerning our research, the techniques we developed to measure movement and its expressive qualities on stage have enabled further degrees of freedom to composers and interactive art artists by allowing real-time control of sound activation and processing through expressive gestures. For example, in Allegoria dell'opinione verbale [Allegory of the Verbal Opinion] (composer R. Doati, 2001), the real-time analysis of the expressive qualities of an actress's lip and facial movements control the sound processing of her voice. In Medea (composer A. Guarnieri, 2002), our EyesWeb platform was used to analyze the movement of trombone players for controlling the 3D spatialization of their sound. In Invisible Line (composer A. Cera, 2007), we sent automatically extracted expressive gesture features over the Internet to make a kind of social and distributed performance in between Genova, Paris, and Hamburg (see www.youtube.com/watch?v=QsPdhO-d4nQ).

Finally, in our ongoing DANCE project (http://dance.dibris.unige.it), the concept of mapping movement onto sound is brought to its extreme consequences - completely inverting the traditional relationship between movement and sound in dance. That is, sound neither inspires body movements in the classical sense nor joins with body movements contingently and accidentally, as in Merce Cunningham's choreographies. Rather, the sound is produced by movement, its qualitative features, and its intrinsic affective and relational meanings. If music is not choreographed, and instead it is the choreography itself that creates music, then dance is conceived as a musical composition (or recomposition and interpretation), changing its traditional dimension into an eminently or exclusively listening experience. Gesture, as an aesthetic object, is experienced as a sounding object. An example of early results from the DANCE project were presented at the SONAR+ Festival in Barcelona in June 2015 (see Figure 3), showing how we developed interactive sonifications of movement qualities such as "fluidity" and "impulsivity."

Figure 3. A snapshot from the booth of the European Horizon 2020 ICT Project DANCE at SONAR+, Barcelona, in June 2015. Short DANCE demos and proof-of-concepts on real-time analysis and interactive sonification of expressive qualities of human movement were developed by the Casa Paganini-InfoMus Research Centre in collaboration with the composer Pablo Palacio and dancers Muriel Romero, Roberta Messa, and Sabrina Ribes. The demo in this picture measures movement qualities using the accelerometers and gyros on two smartphones worn on the dancer's right wrist and left ankle. This initiative was organized by the European Commission Project Officer Ralph Dum in the framework of the European Commission Science, Technology and the Arts (Starts) program. 
The public availability of our EyesWeb platform since 2001 has enabled several collaborations. For example, New York University established a Music, Dance, and New Technologies summer program in Italy from 2003 to 2006. Students in the program learned EyesWeb, used it in their performance, and continued using it afterward. Also, various international centers for artistic research, including IRCAM [Institut de Recherche et Coordination Acoustique/Musique] in Paris and CIANT [International Centre for Art and New Technologies] in Prague, have used EyesWeb in their productions. Furthermore, artists, including visual artists, commonly exploit EyesWeb for their work after learning about the platform from EyesWeb forums or participating in EyesWeb Week, a one-week tutorial we organize every two years at our research center.

We've also developed techniques to measure, in real time, nonverbal social signals among performers, which might provide novel perspectives of the music language and of dance and interactive arts. For example, in the artistic performance tanGO_Touching Music (see www.youtube.com/watch?v=DckQ5XI_B0s), our techniques for analyzing entrainment (that is, temporal and affective synchronization between individuals) and leadership from movement signals captured by mobile devices were exploited to let four dancers and their audience reconstruct a tango. Dancers' quality of movement and nonverbal social signals were used to mold and control the different musical parts of a tango piece.

\section{New Ways of Experiencing Art and Cultural Heritage}

Technology can also influence how art is experienced-for example, how an audience is exposed to works in art installations. According to Italian philosopher Umberto Eco, narration is like a labyrinthine wood in which the reader must walk, continually losing and finding his or her route. Interactive multimedia and multimodal technologies let us implement this intriguing metaphor and further extend it as a new paradigm for actively experiencing artistic content and cultural heritage - that is, technology lets the audience interactively operate the content, modifying and molding cultural objects in real time, in a novel, concrete, and corporeal way. In the late 90s, we started trying to transform such an idea into interactive multimodal installations with work for Città dei Bambini, a science center for children in Genova ${ }^{4}$ and then with work for science centers such as Città della Scienza [Science City] in Naples, the Museum of Balì in Fano, Italy, and the Museum of La Roche d'Oetre in Normandy, France.

More recently, we further developed the concept for an interactive narrative museum, grounded on nonverbal communication, emotional strategies, and empathic, collaborative forms of social interaction. For example, in permanent museum installations for the Enrico Caruso Museum (in Florence, Italy), we developed an interactive environment aimed at recreating an engaging individual or social listening experience of Caruso's voice, starting from the available archives of low-quality audio recordings. The museum's Music Room (see Figure 4) was conceived as an interactive sensitive environment, including a 12-channels 3D interactive audio and loudspeakers system. It lets visitors evoke the artist's voice through queries based on nonverbal full-body movement and behavior. Once a visitor enters the room, each loudspeaker starts simultaneously playing a different fragment of Caruso's voice, subtracted from its harmonic content (so other components of the voice are filtered out). The result is a "whispering" room in which the sound sources surround the visitor. When the visitor moves toward a specific location in the room, a "gush" of a few seconds of the full tenor's voice emerges, related to the content displayed there. Many visitors make several acoustic "gushes" appear, each moving in the room from one visitor to another.

Figure 4. The Music Room at the Enrico Caruso Museum in Florence: (a) visitors experience music "gushes" of the voice of Caruso while exploring the content in the Music Room, and (b) detail of one of the infrared video cameras installed on the ceiling to track the behavior of visitors and control the interactive experience with the voice of Caruso. See also www.youtube.com/watch?v=6GeTsKIFCbE\&list=PLEVgkiAQI8zIO9mMUOcUcQdjA8wLHbFAR.

The interactive museum installation, developed as an EyesWeb application, also analyses in real time the individual and social behavior of visitors, such as a visitor "hesitating in the room" or "remaining still near a content object for a significant amount of time," or the "harmonic relation of the trajectories in the room of each single visitor belonging to a group." These expressive and social features are used to build nonverbal queries, which might result in the retrieval, processing, and then interactive experience of audio fragments from the archive of Caruso's music recordings.

\section{Art-Informed Technologies for Society}

The impact of art-informed scientific and technological research goes far beyond the artistic domain and extends to many other application areas. Consider, for example, our interactive installations Orchestra Explorer and Mappe per Affetti Erranti [Maps for Wandering Affects] ${ }^{2}$ in the framework of our European ICT project SAME. In the Orchestra Explorer installation, a visitor activates and listens to the different instrumental parts of an orchestral score by simply walking on an empty interactive stage, where a virtual orchestra is located. Moreover, the visitor can modify the sound of the instruments with his or her expressive behavior (for example, an aggressive behavior toward a virtual instrument 
"frightens" it). In Mappe per Affetti Erranti, a group of visitors collaborates in reconstructing a music piece in one out of four different pre-recorded expressive performances (joyful, solemn, shy, aggressive) of the same piece (see www.youtube.com/watch?v=V49hiq6R9eY\&list=PLEVgkiAQI8zLU6_ZYEuXW2EOhusFhodcq).

These two installations exemplify how interactive multimedia and multimodal technology, joined with the active experience paradigm, could influence education - particularly music education. They provide a unique way to go deep inside a music piece - in its structure and orchestration and its interpretation by letting visitors simultaneously listen to both the whole piece and a single instrument. The inexperienced user can learn, for example, how to distinguish between the timbre of single instruments, and how the single instruments contribute to the piece as a whole. The user can also learn how altering a single music feature affects the performance and expressive intention it conveys.

In this direction, we recently developed two interactive multimedia applications for children. The Potter application helps children experience and learn sound morphology, while BeSound ${ }^{5}$ helps them learn the basic elements of music composition (see ww.youtube.com/InfoMusLab). Both applications, developed in the framework of the European ICT project MIROR, were evaluated by pedagogues at Goteborg and Bologna University, and BeSound was used in an experimental atelier at a local music school in Belgium, where both teachers and students expressed satisfaction. This research direction is going to continue with a specific focus on violin playing in the new European ICT project TELMI.

Other relevant application domains include therapy and rehabilitation and social inclusion. Research has shown that the sonification of movement can increase body awareness and thus improve the performance and effectiveness of rehabilitation tasks. ${ }^{6}$ Art-informed multimedia and multimodal technology enable and enhance movement sonification toward an aesthetic dimension in terms of an active music experience. We adopted this approach to help patients with Parkinson's disease and, more recently, patients suffering from chronic pain. ${ }^{6}$ In addition to improving rehabilitation performance, the technology also helped foster social inclusion by, for example, reducing the psychological and social barriers experienced by chronic-pain patients.

Our European ICT DANCE project addresses social inclusion. Sonification of choreutic movement investigates forms of sensory substitution ("to see through listening"- that is, to translate from the visual to the auditory domain the expressive qualities of movement), enabling rapprochement and sharing of spaces and emotions between visually impaired and sighted people. Active experience of artistic content can foster social inclusion in complex situations, such as youth deviance.

Furthermore, we recently created, with the Gaslini Children Hospital in Genova, a new joint laboratory called ARIEL (the Augmented Rehabilitation in Interactive/multimodal Environment Lab), where our paradigms, inspired by performing arts and EyesWeb technology, have been adopted for the therapy and rehabilitation of children. Individual as well as social active experience of multimedia content is the core of our current and future research in these and other application scenarios.

Although current ICT might help shape what anthropologist Marc Augé calls the "ideology of the present," entailing the concrete risk of losing our sense of past and history (and therefore our sense of future), ${ }^{7}$ art-informed multimedia and multimodal technologies can help us recover our sense of past. Active experience of musical content brings back to music the embodied and social dimension that analog and digital recording technologies suppressed. Music making and listening are clear examples of human activities that are, above all, interactive and social. Yet to date, these activities have been passive, noninteractive, and noncontext sensitive. Current technologies, with all their potential for interactivity and communication, have not yet been able to support and promote this essential aspect of music making and listening. This can be considered a significant degradation of the traditional listening and music making experience, in which the public was (and still is) able to interact in many ways with performers to modify the expressive features of a music piece.

The integration of techniques to automatically measure, in real time, emotions and nonverbal social signals is a promising direction to boost future technology for the active listening of music and, more in general, the active experience of audiovisual content. Active experience of cultural content brings our society back to its identity, roots, and values by letting us reconnect with the shared language and meaning of our artistic heritage. Art-informed scientific and technological research can enable citizens to rediscover cultural heritage contents in an unconventional and charming way by exploiting interactive, multimedia and multisensory paradigms of narration, combining gestures and words, emotions and cognitions, social and personal retrievals. ${ }^{2}$

\section{Acknowledgments}

The research projects described in this paper are the work of a whole team of researchers at Casa Paganini-InfoMus. We thank our colleagues Paolo Alborno, Corrado Canepa, Paolo Coletta, Nicola Ferrari, Simone Ghisio, Ksenia Kholikalova, Maurizio Mancini, Alberto Massari, Radoslaw Niewiadomski, Stefano Piana, and Roberto Sagoleo. We also thank the European Commission Officers of the projects we coordinated: Loretta Anania (SAME), Teresa De Martino (SIEMPRE), and Ralph Dum (DANCE). We thank the Editor Hayley Hung for her precious suggestions. Note that this research is supported in part by the EU Horizon2020 ICT Project DANCE no.645553. 


\section{References}

1. A.Camurri et al., "EyesWeb-Toward Gesture and Affect Recognition in Dance/Music Interactive Systems," Computer Music J., vol. 24, no. 1, 2000, pp. 57-69.

2. G. Volpe and A. Camurri, "A System for Embodied Social Active Listening to Sound and Music Content," ACM J. Computing and Cultural Heritage, vol. 4, no. 1, 2011, pp. 2-23.

3. N. Bernardini and A. Vidolin, "Sustainable Live Electro-Acoustic Music," Proc. Int'l Sound and Music Computing Conf. (SMC), 2005.

4. A. Camurri and A. Coglio, "An Architecture for Emotional Agents," IEEE MultiMedia, vol. 5, no. 4, 1998, pp. 24-33.

5. G. Varni et al., "Interactive Reflexive and Embodied Exploration of Sound Qualities with BeSound," Proc. 12th Int'l Conf. Interaction Design and Children (IDC), 2013, pp. 531-534.

6. A. Singh et al., "Go-with-the-Flow: Tracking, Analysis and Sonification of Movement and Breathing to Build Confidence in Activity Despite Chronic Pain,” to appear in Human-Computer Interaction, Taylor \& Francis, 2016.

7. M. Augé, Où est passé l'avenir? [Where is the Future?] Editions du Panama, 2008.

Antonio Camurri is a professor at Casa Paganini-InfoMus, which is part of the Department of Informatics, Bioengineering, Robotics, and Systems Engineering (DIBRIS) at the University of Genova. Contact him at antonio.camurri@unige.it.

Gualtiero Volpe is an associate professor at Casa Paganini-InfoMus, which is part of the Department of Informatics, Bioengineering, Robotics, and Systems Engineering (DIBRIS) at the University of Genova. Contact him at gualtiero.volpe@unige.it.

\section{//start sidebar//}

\section{Science Explores the Artistic Process}

Scientific research digs into our neurophysiological, perceptual, cognitive, affective, and social processes to shed light on the creative mechanisms leading an artist to conceive artwork. Consider the following areas:

- $\quad$ ethnographic observation studies how the original creative idea develops and matures;

- $\quad$ experimental psychology investigates how an artwork engages and conveys emotion to an audience;

- $\quad$ social and organizational sciences address how performers collaborate to reach their common goal; and

- $\quad$ neuroscience focuses on the neural mechanisms enabling all of these activities

Recently, such research has benefited from the availability of advanced physical and mathematical models and from sophisticated technological tools providing accurate measures of the phenomena under investigation, thus helping researchers pair qualitative observation with quantitative analysis. For example, social interaction in music ensembles, ranging from small ones (such as string quartets) to sections of orchestras, was studied by applying techniques, such as sample entropy 1 and Granger causality,2 originally conceived to analyze time series in physiology and in econometrics, respectively.

Artistic research benefits in many ways from the increasing body of knowledge and tools science offers it. Whereas the growing knowledge of the mechanisms governing the creative processes might help improve such processes' effectiveness, more importantly, the availability of novel technologies opens formerly unconceivable perspectives and opportunities in terms of extending the artistic language and developing completely new art forms. Consider, for example, dance performances in which the technology extends the choreographic action beyond the dancer's body, coupling it with interactive architectural elements of the environment and with the audience. Or consider novel interfaces for musical expression and collaborative music making, going far beyond the consolidated concept of hyperinstrument, toward novel forms of interaction between instruments, performers, and the audience in distributed environments over the Internet.

\section{References}

1. D. Glowinski et al., "The Movements Made by Performers in a Skilled Quartet: A Distinctive Pattern, and the Function that it Serves," Front. Psychol., vol. 4, 2013, article no. 841.

2. A. D'Ausilio et al., "Leadership in Orchestra Emerges from the Causal Relationships of Movement Kinematics," PLoS One, vol. 7, no. 5, 2012, article e35757. 


\section{Artistic Theories Influence Science and Technology}

Theories and practices coming from artistic research and developed in the context of humanities offer scientific research an alternative perspective for investigating many facets of human behavior. For example, economists have studied social interaction in string quartets to understand the behavior of typical groups of employees (self-managed teams) that have a total responsibility for a defined project in the industrial world.1

Artistic theories provide technology developers with a complementary viewpoint of scientific knowledge to help drive innovation. For example, theories from choreography, such as Rudolf Laban's Theory of Effort,2 have inspired scientific and technological research on automated analysis of human full-body movement as well as on the expressive emotional content movement conveys. Composer Pierre Shaeffer's 3 concept of a sound object and his typomorphology (a kind of taxonomy of sound and its properties) were influential in developing techniques for automatically analyzing sound quality, synthesizing sounds, and controlling sounding objects.

Artistic theories represent a significant theoretical and methodological background for many either consolidated or emerging scientific and technological research domains, such as affective computing, social signal processing, and sound and music computing. Moreover, many companies, especially those in the high-tech field, agree that major skills at the basis of the artistic process, such as creativity and the ability to involve people, are crucial for innovation. (see https://ec.europa.eu/digital-agenda/en/ict-art-starts-platform). Art is thus becoming a valuable catalyst for developing novel value-added products and services for society.

\section{References}

1. A. Gilboa and M. Tal-Shmotkin, "String Quartets as Self-Managed Teams: An Interdisciplinary Perspective," Psychology of Music, vol. 40, no. 1, 2012, pp. 19-41.

2. R. Laban and F.C. Lawrence, Effort, Macdonald \& Evans Ltd.,1947.

3. P. Schaeffer, Traité des Objets Musicaux, Editions du Seuil, 1966.

As art influences science and technology, science and technology can in turn inspire art. Recognizing this mutually beneficial relationship, researchers at the Casa Paganini-InfoMus Research Centre work to combine scientific research in information and communications technology (ICT) with artistic and humanistic research. Here, the authors discuss some of their work, showing how their collaboration with artists informed work on analyzing nonverbal expressive and social behavior and contributed to tools, such as the EyesWebXMI hardware and software platform, that support both artistic and scientific developments. They also sketch out how artinformed multimedia and multimodal technologies find application beyond the arts, in areas including education, cultural heritage, social inclusion, therapy, rehabilitation, and wellness.

Keywords: cross-fertilization, art, artistic research, humanistic research, multimodal technology, healthcare, dance, music, EyesWeb, multimedia, affective computing, mixed reality, human-centered computing, $\mathrm{HCl}$ 